\title{
1 Dynamic evolutionary history and gene content of sex chromosomes across diverse \\ 2 songbirds
}

4 Luo-hao $\mathrm{Xu}^{1,2}$, Gabriel Auer ${ }^{2}$, Valentina Peona ${ }^{3}$, Alexander Suh ${ }^{3}$, Yuan Deng ${ }^{4}$, Shao-hong

5 Feng ${ }^{4}$, Guo-jie Zhang ${ }^{4,5,6}$, Mozes P.K. Blom ${ }^{7}$, Les Christidis ${ }^{8,9}$, Stefan Prost ${ }^{10,11}$, Martin Irestedt ${ }^{7,}$,

6 Qi Zhou ${ }^{1,2, *}$

8 1. MOE Laboratory of Biosystems Homeostasis \& Protection, Life Sciences Institute, $9 \quad$ Zhejiang University, Hangzhou, China

102 2. Department of Molecular Evolution and Development, University of Vienna, Vienna, 11 Austria

3. Department of Evolutionary Biology, Evolutionary Biology Centre, Uppsala University, Uppsala, Sweden

4. China National Genebank, BGI-Shenzhen, Shenzhen, China

5. State Key Laboratory of Genetic Resources and Evolution, Kunming Institute of Zoology, Chinese Academy of Sciences, Kunming, China

6. Section for Ecology and Evolution, Department of Biology, University of Copenhagen, Copenhagen, Denmark

7. Department of Bioinformatics and Genetics, Swedish Museum of Natural History, Stockholm, Sweden

8. National Marine Science Centre, Southern Cross University, Coffs Harbour, NSW, Australia

9. School of BioSciences, University of Melbourne, Parkville, VIC, Australia

10. Department of Integrative Biology, University of California, Berkeley, Berkeley, United States

11. Department of Biology, Stanford University, Stanford, United States

* Correspondence should be addressed to M.I. (Martin.Irestedt@nrm.se) or Q.Z. (zhouqi1982@zju.edu.cn) 


\section{Abstract}

30 Songbirds have a species number almost equivalent to that of mammals, and are classic

31 models for studying mechanisms of speciation and sexual selection. Sex chromosomes are

32 hotspots of both processes, yet their evolutionary history in songbirds remains unclear. To

33 elucidate that, we characterize female genomes of 11 songbird species having ZW sex

34 chromosomes, with 5 genomes of bird-of-paradise species newly produced in this work. We

35 conclude that songbird sex chromosomes have undergone at least four steps of recombination

36 suppression before their species radiation, producing a gradient pattern of pairwise sequence

37 divergence termed 'evolutionary strata'. Interestingly, the latest stratum probably emerged due

38 to a songbird-specific burst of retrotransposon CR1-E1 elements at its boundary, or

39 chromosome inversion on the $\mathrm{W}$ chromosome. The formation of evolutionary strata has

40 reshaped the genomic architecture of both sex chromosomes. We find stepwise variations of Z-

41 linked inversions, repeat and GC contents, as well as W-linked gene loss rate that are

42 associated with the age of strata. Over $30 \mathrm{~W}$-linked genes have been preserved for their

43 essential functions, indicated by their higher and broader expression of orthologs in lizard than

44 those of other sex-linked genes. We also find a different degree of accelerated evolution of Z-

45 linked genes vs. autosomal genes among different species, potentially reflecting their diversified

46 intensity of sexual selection. Our results uncover the dynamic evolutionary history of songbird

47 sex chromosomes, and provide novel insights into the mechanisms of recombination

48 suppression. 


\section{Introduction}

50 Songbirds (Oscines, suborder Passeri) have over 5000 species and comprise the majority of

51 passerines and nearly half of the all extant bird species ${ }^{1}$. This is a result of the largest avian

52 species radiation occurred about 60 million years (MY) ago ${ }^{2}$. Facilitated by the development of

53 genomics, many species besides the zebra finch (Taeniopygia guttata) are now transforming

54 into important models for studying molecular patterns and mechanisms of speciation ${ }^{3,4}$,

55 supergenes ${ }^{5}$ and cognition $^{6}$, out of their long history of ecological or behavioral studies, out of

56 their long history of ecological or behavioral studies. One major reason that has been fueling

57 biologists' fascination with songbirds is their staggering and diversified sexual traits. Many

58 species possess striking plumage forms and colors, sophisticated songs and mating rituals, all

59 of which can undergo rapid turnovers even between sister species. Theories predict that sex

60 chromosomes play a disproportionately large role in speciation (the 'large X/Z' effect), sexual

61 selection and evolution of sexually dimorphic traits ${ }^{7-9}$. However, the evolutionary history of

62 songbird sex chromosome remains unclear, because there were few genomic studies

63 characterizing songbird sex chromosomes except for the Collared Flycatcher (Ficedula

64 albicollis ${ }^{10}$. In contrast to the mammalian XY system, birds have independently evolved a pair

65 of female heterogametic sex chromosomes that are usually heteromorphic in females (ZW) and

66 homomorphic in males (ZZ). A recent cytological investigation of over 400 passerine species

67 found a higher fixation rate of chromosome inversions on the $Z$ chromosome than autosomes

68 within species. Gene flow in the $Z$ chromosome is thus more likely reduced in the face of

69 hybridization ${ }^{11}$. Indeed, a significantly lower level of introgression, and a higher level of Fst in Z-

70 linked genes compared to autosomal genes has been reported from studying pairs of recently

71 diverged songbird species ${ }^{12-15}$. Such a large-Z pattern is probably caused by several factors

72 which act in an opposite manner to the $X Y$ sex system. First, $Z$ chromosomes are more often

73 transmitted in males, thus are expected to have a higher mutation rate than the rest of the

74 genome, due to the 'male-driven evolution' effect ${ }^{16}$. Second, as sexual selection more frequently

75 targets males, the variation in male reproductive success will further reduce the effective

76 population size of $Z$ chromosome from three quarters of that of autosomes ${ }^{17}$. The consequential

77 stronger effect of genetic drift is expected to fix excessive slightly deleterious mutations on the $Z$

78 chromosome, and lead to a faster evolutionary rate than on autosomes (the 'fast- $Z$ ' effect) ${ }^{18}$.

79 This has been demonstrated in the Galloanserae (e.g., chicken and duck) species, those of

80 which undergo strong sperm competition, i.e., more intensive male sexual selection, exhibit a

81 larger difference between the $\mathrm{Z}$ chromosome and autosomes in their evolutionary rates ${ }^{19}$. 
In contrast to the avian $\mathrm{Z}$ chromosome, or more broadly the mammalian $\mathrm{XY}$ chromosomes, the genomic studies of avian W chromosomes, especially those of songbirds have not started only until recently ${ }^{10,20,21}$. This is because most genomic projects prefer to choose the homogametic sex (e.g., male birds or female mammals) for sequencing, in order to avoid the presumably gene-poor and highly repetitive $\mathrm{Y}$ or $\mathrm{W}$ chromosomes. The $\mathrm{Y} / \mathrm{W}$ chromosomes have undergone suppression of recombination to prevent the sex-determining gene or sexually antagonistic genes (beneficial to one sex but detrimental to the other) from being transmitted to the opposite $\operatorname{sex}^{22}$. As a result, interference between linked loci ('HillRobertson' effect) reduces the efficacy of natural selection and drives the ultimate genetic decay of non-recombining regions of $\mathrm{Y} / \mathrm{W}$ chromosomes ${ }^{23}$. This process can be accelerated by positive selection targeting, for example, male-related genes on the $\mathrm{Y}$ chromosome ${ }^{24}$; or by

93 background selection purging the deleterious mutations from highly dosage-sensitive genes ${ }^{25}$.

94 Simulation showed that both forces play a different role at different stages of $Y / W$ 95 degeneration ${ }^{26}$. Both have been implicated in analyses of mammalian ${ }^{24,27}$ and Drosophila $^{28,29} \mathrm{Y}$ 96 linked genes. However, no evidence has been found for female-specific selection among the Wlinked genes (also called gametologs) of chicken ${ }^{21}$ or flycatcher ${ }^{30}$. latifolia $^{32}$ ), recombination suppression has proceeded in a stepwise manner presumably through chromosome inversions, leaving a stratified pattern of sequence divergence between sex chromosomes termed 'evolutionary strata' ${ }^{\prime 3}$. Eutherian mammalian $\mathrm{X}$ and $\mathrm{Y}$ chromosomes have been inferred to share at least three strata, with another two more recent ones shared only among catarrhines (old world monkeys and great apes) ${ }^{27}$. It has been recently discovered that the history and tempo of avian sex chromosome evolution is much more complicated than that of mammals ${ }^{20}$. All bird sex chromosomes only share the first step of recombination suppression (stratum 0, Aves S0) encompassing the avian male-determining gene DMRT1. This was

107 followed by the independent formation of $S 1$ in the Palaeognathae (e.g., ratites and tinamous)

108 and in the ancestor of the Neognathae (all other extant avian radiations). Ratites have halted

109 any further recombination loss and maintained over two thirds of the entire sex chromosome 110 pair as the exceptionally long recombining pseudoautosomal regions (PAR). Therefore, their W 111 chromosomes are unusually homomorphic and gene-rich comparing to the Z chromosomes. In 112 contrast, all species of Neognathae examined have suppressed recombination throughout most 113 regions of the sex chromosomes with short and varying sizes of PAR $^{34}$. Overall, avian $\mathrm{W}$ 114 chromosomes seem to have retained more genes and decayed at a slower rate than the 115 mammalian $\mathrm{Y}$ chromosomes. Furthermore, sexually monomorphic species (e.g., most ratites) 
116 seem to differentiate even slower than sexually dimorphic species (chicken and most Neoaves)

117 in their sex chromosomes, consistent with the hypothesis that sexually antagonistic genes have

118 triggered the expansion of recombination suppression between sex chromosomes ${ }^{35}$. However,

119 due to the ratites' deep divergence from other birds, and also an expected much lower mutation

120 rate due to their larger body size and longer generation time, it is unclear what the actual

121 influence of sexual selection is on the rate of sex chromosome evolution. All Neoaves species

122 share one stratum S2, with the more recent evolutionary history of sex chromosomes of

123 songbirds unclear. So far, only one songbird, the collared flycatcher has been extensively

124 characterized for its $\mathrm{W}$-linked genes ${ }^{30}$, whose number is within the range of 46 to $90 \mathrm{~W}$-linked

125 genes reported for other Neoaves ${ }^{20}$. To elucidate the evolutionary history of songbird sex

126 chromosomes, we produced high-quality female genomes of five birds-of-paradise (BOP).

127 Together with a re-analysis of 6 other published female genomes of songbird species ${ }^{30,36-39}$, our

128 analyses cover the two major songbird lineages (Corvida and Passerida) that rather diverged in

129 the last $50 \mathrm{MY}^{2,40}$.

\section{Results}

\section{Characterization of songbird sex chromosome sequences}

133 We produced between 36-150 fold genomic coverage of sequencing data for each BOP species,

134 and performed de novo genome assembly followed by chromosome mapping using the great tit

135 (Parus major) genome as reference ${ }^{6}$. The high continuity and completeness of the draft

136 genomes are reflected by their scaffold N50 lengths (all >3 Mb except for the raggiana BOP,

137 Paradisaea raggiana) and BUSCO core gene completeness scores (92.9\% to 94.0\%)

138 (Supplementary Table 1). To reconstruct the evolutionary history of sampled songbird sex

139 chromosomes, we first identified the sex-linked sequences from the draft genome of each

140 species. We searched for the scaffolds from sexually differentiated regions (SDR) as those that

141 show half the female sequencing depth of autosomes (Fig. 1A, Supplementary Fig. 1).

142 Scaffolds from putative PARs were inferred by their homology to that of flycatcher ${ }^{41}$, and also

143 sequencing depth similar to autosomes. It is noteworthy that our method cannot identify very

144 recent fusion/translocation of autosomal fragments to the sex chromosome pair ('neo-sex'

145 chromosome), as in the case of warblers ${ }^{42}$. All the studied songbirds have a short putative PAR

146 ranging from $564 \mathrm{~kb}$ to $781 \mathrm{~kb}$. We then categorized SDR sequences as either Z- or W-linked

147 with the expectation that the $\mathrm{W}$ chromosome would diverge much faster than the homologous $Z$

148 chromosome of the same species, when being compared to the $Z$ chromosome of an outgroup

149 species (Materials and Methods), as a result of rapid accumulation of deleterious mutations 
and repetitive elements after recombination suppression. It is possible that Z-linked paralogs, although not expected to be abundant in the compact avian genomes ${ }^{36}$, would confound our identified W-linked sequences by showing a similar sequence divergence pattern. Thus, among the five species with male sequencing data, we further verified the sex-linkage by confirming all the putative W-linked scaffolds with a clear female-specific pattern (Fig. 1A, Supplementary Fig. 2). The assembled lengths of the largely euchromatic parts of $W$ chromosomes range from 1.33 to $6.52 \mathrm{Mb}$, corresponding to only $1.9 \%$ to $8.5 \%$ of the $\mathrm{Z}$ chromosome length across species (Fig. 1B, Supplementary Table 2), probably as a result of large deletions and massive invasions of repetitive elements which might be difficult to assemble with existing technologies. Indeed, the repeat content of the assembled W chromosomes is 2.5 to 4.9 fold higher than that of $\mathrm{Z}$ chromosomes on the chromosome-wide average (Supplementary Fig. 3 and Table 2), consistent with the patterns of $W$ chromosomes of chicken and flycatcher ${ }^{43,44}$. We have not found shared syntenic W-linked regions across species that may have been deleted in the ancestor of songbirds compared to the chicken or ostrich genome, as suggested by previous cytological studies ${ }^{45}$.

\section{Age-dependent genomic impact of evolutionary strata}

If recombination was suppressed between sex chromosomes in a stepwise manner, we expect

to find a gradient of Z/W sequence divergence levels along the chromosome sequence of the $Z$ chromosome, like what has been reported along the human $\mathrm{X}$ chromosome ${ }^{46}$. Previous work showd that the $Z$ chromosomes of the Neognathae have undergone dramatic intrachromosomal rearrangements which resulted in a misleading extant synteny for ordering different evolution strata $^{20}$. By contrast, the Palaeognathae (e.g. emu Dromaius novaehollandiae and ostrich Struthio camelus) have maintained highly conserved sequence synteny even with reptile species, with over two thirds of their sex-linked regions still recombining as an appropriate approximate of proto-sex chromosomes of all bird species ${ }^{20}$. We first confirmed these patterns by comparative mapping of the nearly chromosome-level assemblies of $Z$ chromosomes of collared flycatcher, hooded crow (Corvus corone) ${ }^{47}$ vs. chicken and emu (Fig. 2A). This allows us to reconstruct the spanned regions of $S 0$ shared by all birds, and a part of $S 1$ shared by all Neognathae species in the studied songbird genomes by their homology to the emu genome. They are mapped as two continuous regions on the emu $Z$ chromosome, but have become severely reshuffled into dispersed fragments in songbirds (Fig. 2A, Supplementary Fig. 4-5). Two recently formed strata (Neoaves S2 and S3) are highly conserved for their synteny across avian species, and each show significantly $(P<0.05$, Wilcoxon test $)$ different levels of Z/W 
sequence divergence (Fig. 2B, Supplementary Fig. 6), GC3 (GC content at the third codon positions, Supplementary Fig. 7) and Z-linked long terminal repeat (LTR) content (Fig. 2C, Supplementary Fig. 7) from each other. The drastic change of Z/W sequence similarity allows us to precisely map the boundaries between these two strata. In general, a series of recombination suppression has reshaped the genomic architecture of $Z$ chromosomes in a chronological order. Regions of younger strata exhibit much fewer Z-linked intrachromosomal rearrangements between species, suggesting the reduced selective constraints on gene synteny after recombination was suppressed in the older strata ${ }^{48}$. In particular, GC3 content decreases, while the repeat content increases by the age of stratum. This is probably because of weaker effects of GC-biased gene conversion ( $\mathrm{gBGC})^{49}$, and purifying selection against TE insertions ${ }^{50}$ under reduced recombination, both of which have been acting for a longer time within Z-linked regions of older strata. Consistently, a similar pattern has also been found contrasting PAR vs. the remaining Z-linked regions in the collared flycatcher ${ }^{41}$.

\section{Lineage-specific burst of retrotransposon probably has induced recombination} suppression between sex chromosomes

The distribution of long interspersed nuclear elements (LINEs), mainly the retrotransposon chicken repeat 1 (CR1) elements shows an exceptional pattern compared to that of LTR elements (Fig. 2C, Supplementary Fig. 7) associated with the age of strata. S3 presents a similar proportion of CR1 with S0 that is much higher than the rest of the Z-linked regions. A close examination shows that this is due to the specific accumulation of CR1 elements at the boundary between PAR and S3. Such a burst of CR1, particularly only the CR1-E1 subfamily ${ }^{51}$, extends into about one third of the entire S3 region and is shared by all investigated songbirds but absent in the deep-branching passerine rifleman (Acanthisitta chloris) and other Neoaves to such accumulation of CR1-E1, and also previously reported multiple deletions in passerines that removes a gene $D C C$ (Deleted in Colorectal Carcinoma) highly conserved across other vertebrates $^{52}$. This gene is responsible for axon guidance for brain midline crossing and has independently been lost in some but not all passerines and Galliformes ${ }^{53}$.

In addition, we find evidence that the burst of CR1-E1 elements coincides with the S3

214 emergence. Our phylogenetic reconstruction of Z- and W-linked gametolog sequences shows

215 that songbird-derived sequences are always grouped by chromosome instead of species,

216 compared to outgroup birds (Fig. 2E, Supplementary Fig. 9-12). This indicates that all

217 songbirds share four evolutionary strata, with the latest stratum S3 formed after the divergence 
218 between songbirds and the rifleman. The highly conserved synteny between songbird species,

219 and between songbirds and chicken of S3 on the Z chromosome (Fig. 2A, Supplementary Fig.

220 5), suggests that there was no chromosomal inversion on the $Z$ chromosome at S3, and the

221 recent burst of the CR1-E1 subfamily probably contributed to the formation of S3, although we

222 cannot exclude the contribution of possible W-linked chromosomal inversions. Interestingly,

223 other CR1 subfamilies (CR1-E4, CR1-E5, CR1-E6) have an independent burst at the PAR/S3

224 boundary in rifleman (Fig. 2D, Supplementary Fig. 8 and Table 3). Given that this boundary

225 region has been shown to have frequent but different degrees of multiple gene loss in different

226 lineages of birds ${ }^{52,53}$, it is very likely a hotspot for structural changes (including LINE

227 accumulation) that have recurrently contributed to the independent formation of S3 in many bird

228 species.

\section{Fast-Z pattern of songbirds implies dynamic evolution of sexual selection}

The formation of evolutionary strata has subjected the $Z$ chromosome to male-biased transmission and a reduced effective population size, which are expected to produce faster mutation and evolutionary rates of Z-linked genes ${ }^{17}$. Indeed, we found a larger branch-specific synonymous substitution rate (dS) of Z-linked genes (statistically not significant), but a significantly smaller dS of W-linked genes, compared to that of autosomal genes $(P<0.01$, Wilcoxon rank sum test, Supplementary Fig. 13), as a result of male-driven evolution. The branch-specific evolutionary rates $(\omega)$ measured by the ratios of nonsynonymous substitution rate $(\mathrm{dN})$ over dS have significantly $(P<0.01$, Wilcoxon rank sum test, Supplementary Fig. 14) increased for both Z- and W-linked gametologs relative to autosomal genes, indicating a 'fast-Z' effect and degeneration of W-linked genes (see below). Previous simulation work and experimental evidence in Galloanserae have suggested that different degrees of sexual

242 selection targeting males will influence the male-mating success, hence the genetic drift effect

243 on the $Z$ chromosome to a different degree ${ }^{17,19}$. Songbirds, especially BOPs, have been

244 frequently used as textbook demonstrations for sexual selection, though the long-term

245 evolutionary history of sexual selection remained unclear. To reconstruct this, we approximate

246 the intensity of sexual selection targeting males by measuring the fast- $Z$ effect ( $Z / A$ value, the

247 ratio of branch-specific $\omega$ values of Z-linked genes vs. autosomal genes) in a phylogenetic

248 context (Fig. 3, Supplementary Table 4). The varying Z/A values at different lineages suggest

249 a dynamic change of intensity of sexual selection, even among the five BOP species that

250 diverged within the last $15 \mathrm{MY}^{54}$. While the significant (permutation test, $P<0.05$ ) fast-Z pattern

251 of the sexually monochromatic American crow (Corvus brachyrhynchos) may reflect the sexual 
selection acting on the ancestral lineage leading to the Corvidae (crows, jays and allies), a lack of such a pattern in the raggiana BOP and magnificent BOP (Cicinnurus magnificus) is somewhat unexpected. These species are known for their lekking behaviors ${ }^{55}$, with which very few males dominate almost all females for copulation through outcompeting other males with displays. This produces a strongly biased male-mating success, and direct challenge for maintaining genetic variation in the population ('the lekking paradox') ${ }^{56}$. Very few field quantitative studies have been performed on BOP species, and it will be interesting to investigate whether raggiana and magnificent BOPs female individuals may solve the 'lekking paradox' by changing their mating preference, and in fact mate with more males than is presumed.

\section{Conserved gene content of the songbird W chromosomes}

In contrast to the dynamic evolution of Z-linked genes and sequences, W chromosomes of all the studied songbirds have undergone dramatic gene loss but exhibit an unexpected conservation of the retained gene repertoire across species. The numbers of assembled Wlinked genes range from 31 in house sparrow (Passer domesticus) to 63 in the king BOP (Cicinnurus regius), compared to about 600 to 800 Z-linked genes in each species (Fig. 4A, embedded in highly repetitive regions maybe missing from the current $\mathrm{W}$ chromosome assemblies. In general, Corvida species retain more W-linked genes than Passerida species

272 (Supplementary Table 5), likely due to their longer generation time thus a lower mutation rate. Most W-linked genes are single-copy without lineage-specific expansion, except for HINT1W (Supplementary Fig. 15). Despite the relative rarity of gene duplication in birds compared to mammals ${ }^{57}$, we find one $\mathrm{W}$-linked gene that is a duplicated copy from an autosomal gene NARF in American crow (Supplementary Fig. 16). This duplicated gene is also present in another Corvida species Lycocorax pyrrhopterus (Alexander Suh, personal communication), suggesting that the duplication event likely occurred in the ancestor of Corvida. It will be interesting to see whether this gene shows signatures of female-specific selection, e.g, novel patterns of ovaryspecific expression, which drives its fixation on the W. Fifty-seven genes are shared by at least one Corvida and another Passerida species, and 23 genes are shared between at least one songbird species and the chicken ${ }^{21}$. This suggests they were still preserved on the $\mathrm{W}$

283 chromosome before the divergence of passerine or Neognathae species. Interestingly, despite 284 the independent origin of S2 in the chicken and Neoaves ${ }^{20}$, all the chicken W-linked genes but 285 one are also found in passerines, indicating similar underlying evolutionary forces governing 
their convergent retention since Galloanserae and Neoaves diverged from each other $89 \mathrm{MY}$ ago.

To examine such forces, we perform gene ontology (GO) analyses on the 79 genes that are present on the $\mathrm{W}$ chromosome of at least one songbird species. They are enriched $(P<$ 0.01 , Fisher's exact test) for two GO terms of 'DNA binding' and 'transcription factor activity, sequence-specific DNA binding' (Supplementary Table 6). This suggests that similar to the mammalian Y-linked genes ${ }^{27}$, some W-linked genes are retained for their important functions of regulating gene activities elsewhere in the genome. The Z-linked homologs of lost genes evolve significantly $(P<0.01$, Wilcoxon rank sum test) faster with their $\omega$ ratios higher than those of the retained genes on the $\mathrm{W}$ chromosome (Fig. 4B, Supplementary Fig. 17). This shows a different selective pressure acting on these two sets of genes on the proto-sex chromosomes. As this pattern maybe confounded by the 'faster-Z' effect of hemizygous Z-linked genes, we further study the autosomal orthologs of these genes in the green anole lizard (Anolis carolinensis). We find that the orthologs of retained genes have significantly $(P<1.497 \mathrm{e}-05$, Wilcoxon rank sum test, Fig. 4C) higher expression levels in all lizard tissues of both males and females, and also a broader tissue expression pattern than those of the lost genes across all the tissues (Fig. 4D). The patterns are also consistent among the four songbird evolutionary strata. These results are robust if we use emu to infer the ancestral expression pattern (Supplementary Fig. 18), whose sex chromosomes are largely a PAR. Consistent with the result of collared flycatcher ${ }^{10}$, we find no evidence that female-specific selection may prevent the gene loss, or drive certain genes to undergo positive selection on the songbird $\mathrm{W}$ chromosomes. We found no excess of ovary-biased lizard orthologs among those of the retained W-linked genes: only 6 out of $72(8.3 \%)$ are ovary-biased while the genome-wide proportion is about $20 \%$.

312 Overall, $4.6 \%$ to $9.2 \%$ of single-copy W-linked genes per songbird species (Fig. 4A), compared

313 to $1.6 \%$ to $3.0 \%$ single-copy $Y$-linked genes per mammalian species ${ }^{27}$ have been retained for

314 their essential or sex-specific functions. A seemingly high retention ratio of $\mathrm{W}$-linked genes in

315 birds can be partially attributed to the generally much lower mutation rate of W chromosome 316 relative to $\mathrm{Y}$ chromosome by male-driven evolution effect (Supplementary Fig. 13), assuming a

317 similar generation time between mammals and birds. In addition, a much more frequent and

318 stronger sex-specific selection acting on the $\mathrm{Y}$ chromosome than on the $\mathrm{W}$ chromosome,

319 sometimes driving the massive expansion of $Y$-linked gene copies with male-related function ${ }^{24}$, 
320 probably also has contributed to a faster rate of $Y$ chromosome gene loss by the hitchhiking

321 effect $^{24,26}$. To scrutinize the tempo of gene loss throughout the evolution of songbird sex

322 chromosomes, we conservatively reconstructed the numbers of retained $\mathrm{W}$-linked gametologs

323 at each phylogenetic node of the avian tree (Fig. 5A, Supplementary Table 8), by identifying

324 the genes present on any of the studied avian W chromosomes. We find that within each

325 stratum, the percentage of gene loss is always much larger at an earlier evolutionary time point

326 than in the recent ones, and this is consistent between birds and mammals (Fig. 5B). Thus the

327 majority of gene loss has probably occurred during the early stage of $\mathrm{W}$ or $\mathrm{Y}$ chromosome

328 evolution, and the rate of gene loss dramatically decreases by the less retained genes. Although

329 convergent gene loss may cause an overestimation of lost genes at more ancestral time points

330 (e.g., in S0 region), this probably has little influence on our estimate in the most recent songbird-

331 specific stratum S3 which has already lost $69.8 \%$ of the W-gametologs within $50 \mathrm{MY}$. We also

332 find that the retained genes of songbird $W$ chromosomes are often close to each other

333 (Supplementary Fig.19), suggesting large sequence deletions have had an important

334 contribution to drastic gene loss.

The decrease of gene loss rate on Y/W chromosomes through evolutionary time can be explained by a weaker Hill-Robertson effect that the less retained genes can induce, which has been previously shown in a simulation study ${ }^{26}$. Thus, the ancestral gene number of older evolutionary strata which would have undergone more serious gene loss must have a larger influence on the extant number of retained genes. A lower rate of retained mammalian Y-linked genes relative to avian $\mathrm{W}$-linked genes, besides the cause of male-driven evolution, can be also attributed to the fact that the first two or three mammalian evolutionary strata emerged before the divergence of eutherians which together account for over $93.2 \%$ of the entire gene content of ancestral $Y$ chromosome, while those of birds only account for $53.3 \%$ of the entire ancestral W-linked gene content (Fig. 5C).

347 The evolution of sex chromosomes is usually, but not always (e.g., in frogs ${ }^{58}$, ratite birds ${ }^{20}$ and 348 python ${ }^{59}$ ), marked with episodes of recombination suppression that eventually restricts the 349 recombining region to one or two small PARs at the end of the sex chromosomes. The resulting 350 patterns of evolutionary strata have been widely reported in many animal and plant species, 351 with the responsible formation mechanism presumed to be chromosomal inversions ${ }^{60}$. Indeed, 352 footprints of inversions in the latest two strata between mammalian $X$ and $Y$ chromosomes have 353 been found by examining the synteny order between $X / Y$, and particularly the $X$-linked boundary 
sequence that has been disrupted into two dispersed sequences on the $Y$ chromosome ${ }^{61}$. In songbirds, we show here that four evolutionary strata were formed before their species radiation. Importantly, we provide evidence suggesting an alternative scenario of recombination suppression. We find in the latest stratum S3, a genome-wide burst of a certain transposable element (TE) led to its specific accumulation at the mutation/insertion hotspot near the PAR boundary of the sex chromosomes (Fig. 2, Supplementary Table 3), which probably further mediated recombination suppression. It has been reported with some exceptions, in many species that local recombination rates and abundance of TE elements generally have a negative association, with their causal relationship difficult to be disentangled ${ }^{50}$. In the case of the songbird S3 region, several patterns suggest that TE accumulation might be the cause rather than the result of recombination suppression. First, in several species (e.g., Lawes's parotia (Parotia lawesi) and the king BOP, Fig. 2D, Supplementary Fig. 8), the responsible CR1-E family is also enriched close to the PAR boundary, where normal levels of recombination can be expected. Second, the abundance of CR1-E gradually decreases further away from the boundary, which also has undergone genomic deletions or rearrangements independently in other bird species ${ }^{52,53}$, thus is likely to be a mutation hotspot. Third, only CR1-E repeats, but not any other type of CR1 or repeat families, are enriched at the PAR/S3 boundary. These results are not likely to be expected if S3 stratum formation occurred before the CR1-E accumulation, which predicts a uniformly distributed accumulation of various kinds of repeat elements (e.g., LTR elements, Supplementary Fig. 20) by Hill-Robertson interference effects that would not extend into the PAR. Indeed, our comparative analyses between species suggest no Z-linked inversion in S3 (Fig. 2A, Supplementary Fig. 5), though we cannot exclude the possibility of a $\mathrm{W}$-linked inversion that may have instead contributed to the formation of S3. The verification of the latter requires future improvements of genome assembly using for example, PacBio/Nanopore sequencing technology to assemble the highly repetitive W-linked sequence.

Under our proposed scenario, TEs probably reduced the recombination rate in PAR through, for example, changing the chromatin structure or disrupting recombination hotspots ${ }^{50}$. The CR1-E retrotransposon accumulation occurred at the mutation hotspot located at PAR/S3

382 boundary despite the selection against its deleterious effects of disrupting gene functions. This 383 has resulted in partial or complete deletion of several genes in songbirds ${ }^{52,53}$. However, the 384 consequential reduction of recombination rate can provide the selective advantage of 385 accelerating the fixation of pre-existing sexually antagonistic (SA) alleles in PAR through sex386 biased transmission; or subjecting the PAR for the 'fast-Z' evolution by male-driven evolution 387 effect (Fig. 3) and increasing its exposure for male-biased selection, so that novel SA alleles 
may more frequently emerge and become fixed. The latter has been implicated by the recent findings in songbirds that male-specific trait genes, for example those related to sperm morphology ${ }^{62}$ or plumage colors ${ }^{63}$, which have recently become diverged within or between populations, are enriched on the $Z$ chromosome. In addition, TE accumulation is likely to increase the chance of chromosome inversions through ectopic recombination, or by reducing the selective constraints on gene synteny ${ }^{48,50}$. The latter is supported by our result that older evolutionary strata have undergone more Z-linked genomic rearrangements between songbird species than the younger ones (Fig. 2A), which creates a positive feedback once the recombination suppression was initiated. This provides a mechanistic explanation for the more frequent fixation of Z-linked inversions found among passerines.

While the $\mathbf{Z}$ chromosome is predicted to accumulate dominant male-beneficial mutations, the $\mathrm{W}$ chromosome is expected to accumulate female-beneficial mutations responding to female-specific transmission ${ }^{64}$. Here we find that more genes to have been retained on the $\mathrm{W}$ chromosomes of songbirds than that of chicken (Fig. 4). However, both previous works on the chicken and collared flycatcher ${ }^{21,30}$, as well as our present study, have not found evidence for such 'feminization' of W chromosome. This is in contrast to multiple reported cases of 'masculinization' of the $\mathrm{Y}$ chromosome in the ancient mammalian $\mathrm{Y}$ chromosome systems ${ }^{24}$ or the recently-evolved $\mathrm{Y}$ chromosome of Drosophila miranda ${ }^{28}$. Genes specifically expressed in the male germline have either massively amplified their copy numbers, or upregulated their expression levels on these evolving $Y$ chromosomes. Such a difference can be explained by the fact that regardless of the sex chromosome type, sexual selection is often targeting males in most species, thus the $Z / Y$ chromosomes are more frequently influenced than the $W / X$ chromosome due to their male-biased transmission, although $X$ chromosomes are nevertheless expected to accumulate recessive male-beneficial alleles ${ }^{9}$. The convergently evolved pattern shared between the mammalian $\mathrm{Y}$ and avian $\mathrm{W}$ chromosomes is largely attributed to the

413 essential genes that have important regulatory functions and are preferentially retained over the 414 long period of recombination suppression (Fig. 4) ${ }^{21,27}$. However, previous transcriptome 415 comparison of chicken breeds selected for egg-laying vs. fighting, i.e. female-specific vs. male416 specific traits, has found that most W-linked genes are upregulated in the former ${ }^{64}$. Few high417 quality avian W chromosome sequences are available except for that of chicken ${ }^{21,65}$. Songbirds 418 provide a rich resource with many species having a reversed direction of sexual selection and 419 ornamented females ${ }^{66}$. Application of long-read sequencing technology in future will help to 420 elucidate the role of the $\mathrm{W}$ chromosome in sexual selection and speciation of birds ${ }^{67}$. 


\section{Materials and Methods}

424 Genomic DNA were extracted from fresh female tissue samples of from BOP species

425 Cicinnurus regius (ANWC B24969), Cicinnurus magnificus (ANWC B27061), Paradisaea rubra

426 (YPM84686), Parotia lawesii (ANWC B26535), using Thermo Scientific ${ }^{\text {TM }}$ KingFisher $^{\text {TM }}$ Duo

427 Prime purification system. Paired-end and mate pair libraries for these samples were prepared

428 by SciLifeLab in Stockholm, Sweden. All libraries were sequenced on Illumina HiSeq 2500 or

429 Hiseq X v4 at SciLifeLab in Stockholm, Sweden. For Paradisaea raggiana (USNM638608),

430 genomic DNA were extracted using EZNA SQ Tissue DNA Kit. One paired-end and one mate-

431 pair libraries were sequenced on Illumina HiSeq 2000. We also used published female genomes

432 of Corvus brachyrhynchos, Serinus canaria, Passer domesticus, Geospiza fortis, Ficedula

433 albicollis, Pseudopodoces humilis for analysis in this work (Supplementary Table 9) ${ }^{30,36-39}$. The

434 BOP genomes were assembled using ALLPATHS-LG ${ }^{68}$ with 'HAPLOIDIFY=True'. For $P$.

435 raggiana, due to the lack of overlapping paired-end reads, SOAPdenovo $2^{69}$ was used instead.

436 Gene models were annotated using the MAKER pipeline in two rounds ${ }^{70}$. The reference protein

437 sequences of zebra finch, great tit, hooded crow, American crow, collared flycatcher and

438 chicken were downloaded from NCBI RefSeq (Supplementary Table 9). Using the reference

439 protein sequences and chicken HMM (Hidden Markov Models), an initial set of gene models

440 was obtained by using MAKER, and those models were taken for SNAP model training ${ }^{71}$. In

441 addition, 3000 gene models were selected for Augustus training ${ }^{72}$. The trained gene models and

442 the protein sequences were taken as input for MAKER in the second run. To annotate repeats,

443 first we used RepeatModeler ${ }^{73}$ to identify and classify repeat elements for each species,

444 including the published genomes ${ }^{74}$. Then we combined each individual library with an avian

445 repeat library and further manually curated BOP repeat consensus sequences, followed by

446 annotation repeats using RepeatMasker $(\mathrm{v} 1.7)^{73}$.

\section{Identification of sex-linked sequences}

449 We used the sequence of the great tit Z chromosome as reference to search for homologous Z-

450 linked sequences in the studied species, as it has the best assembly quality (contig N50: 133kb,

451 scaffold N50: 7.7Mb) among the published genomes of songbirds ${ }^{6}$. We used nucmer from

452 MUMMer package (v4.0) ${ }^{75}$ for genome-wide pairwise sequence alignment. For any scaffold

453 larger than $10 \mathrm{~kb}$ that has more than $60 \%$ of the sequence aligned to great tit chrZ, it was

454 identified as candidate Z-linked sequences. They were further inspected by comparing the

455 female sequencing coverage to that of autosomes. To do so, the raw female reads were 
mapped to the genome references by using bwa ${ }^{76}$ then the mean sequencing depth of every 50 $\mathrm{kb}$ window was calculated. Sequencing depth for single sites was counted using 'samtools depth' before calculating window-based coverage. Any site with low mapping quality (less than $60)$ and very high coverage (3 times larger than average) was excluded.

To identify the $\mathrm{W}$-linked scaffolds, first we identified the scaffolds that show halfcoverage relative to that of autosomes. We plotted the distribution of coverage of all scaffolds to decide the cutoff of 'half-coverage' (Supplementary Fig.1). Those half-coverage scaffolds were 463 expected to be either Z-linked or W-linked. To distinguish between the Z and W, we aligned the 464 half-coverage scaffolds to the $Z$ chromosome from the hooded crow, a closely related species to 465 BOP, whose genome is derived from a male without contamination of W-linked sequences. We 466 used nucmer for sequence alignment and only kept 1-to-1 best alignments. We then calculated 467 the proportion of sequences of each scaffold that was aligned to the hooded crow chrZ, and 468 decided a cutoff to separate the $Z$ and $W$ based on the distribution of the proportion of Z-linked 469 alignment. We further excluded the candidate W-linked scaffolds that over $10 \%$ of the sequences were aligned to hooded crow autosomes, or a larger portion of sequences aligned to the $\mathrm{Z}$ chromosome than the W. Finally, only the scaffolds that were larger than $50 \mathrm{~kb}$ were kept. We also retrieved additional Z-linked scaffolds that were absent in the results from the homology-based approach, likely due to the missing Z-linked sequences in the great tit Z chromosome assembly. For ground tit, medium ground finch, house sparrow and collared flycatcher in which both male and female sequencing reads are available, the W-linked sequences were further verified by mapping the male reads. Specifically, for every scaffold, the number of nucleotide sites that were covered by male and female sequencing data were counted respectively as $\mathrm{Nm}$ and $\mathrm{Nf}$, and the ratios of $\mathrm{Nm}$ to $\mathrm{Nf}$ were calculated. W-linked scaffolds were expected to have $\mathrm{Nm} / \mathrm{Nf}$ ratios close to zero and one for autosomal or Z-linked scaffolds. For PARs, we used the known sequences of the zebra finch $^{77}$ and collared flycatcher ${ }^{41}$ to search for the homologous sequences in other species using nucmer. The female sequencing depths of those candidate PARs were compared to autosomes and required to be similar.

\section{Demarcation of evolutionary strata}

486 We ordered and oriented the identified Z-linked scaffolds into one pseudo-chromosomal 487 sequence (pseudo-chrZ) based on their alignments against the chromosomal assembly of great 488 tit. Hooded crow has only 15 Z-linked scaffolds and 10 out of them are larger than $1 \mathrm{Mb}^{47}$, thus 489 was used as a representative Corvida species for comparison on the $Z$ chromosome. We 
490

\section{2}

determined the relative order and orientation of the scaffolds according to their alignment on the great tit Z chromosome. Similarly, for BOP species, we created pseudo-chromosome Z using great tit as guiding reference. The pseudo-Z chromosome of emu was built using ostrich $Z$ chromosome ${ }^{78} 79$ as reference. We used nucmer for pairwise alignment of the $Z$ or pseudo- $Z$ chromosomes. Alignments short than $2 \mathrm{~kb}$ were excluded.

The W-linked scaffolds were then aligned to the pseudo-chrZ using last $z^{80}$, after masking repetitive sequences. Sequence similarity of the alignments between the chrZ and chrW was calculated by the script psIScore from UCSC Genome Browser (https://genome.ucsc.edu/). Individual alignments that had sequence similarity lower than 60 or higher than 96 , or alignment length shorter than 65 were removed. After that, we concatenated alignments within nonoverlapping sliding windows of $100 \mathrm{~kb}$, and calculated sequence similarity for the concatenated alignments. When the length of concatenated alignments was shorter than $2 \mathrm{~kb}$ within a 100-kb window, the window was excluded from further analyses. The window-based sequence similarity was then plotted along the pseudo-chrZ. The shift of sequence similarity was used to demarcate the boundaries of S3/S2 and S2/S1. Since very few W-linked sequences have been assembled for the most ancient stratum S0, we mapped its reshuffled fragments in songbirds based on their homology with the emu S0. Our previous study showed emu has a recent species-specific stratum (S1) while the first stratum (S0) is ancient and shared by all birds ${ }^{20}$. This allows for the demarcation of S1 and S0 by detecting their differential degree of ZW differentiation. Specifically, by using relatively relaxed mapping criteria (bwa mem) to map female sequencing reads, only SO showed reduced coverage relative to autosomes or PAR (Supplementary Fig. 4), while S1 showed reduced coverage when stringent mapping was applied (bwa sampe -a $900-\mathrm{n} 1-\mathrm{N} 0-0$ 10000).

To scrutinize the accumulated LINE (mostly CR1) elements at the PAR/S3 boundary, we first divided them into each subfamily (approximated by each repeat consensus sequence) according to the RepeatMasker annotation. Among all subtypes, CR1-E1 is usually ranked with the highest or second highest number at the $S 3$ region across all songbird species. Other highranking subtypes included CR1-E3, CR1-E5, CR1-E4, CR1-E6, CR1-J2 and CR1-Y2. Then we plotted each subtype's abundance with a $100 \mathrm{~kb}$ non-overlapping window along the $Z$ chromosome, in all the studied songbirds, as well as outgroup species rifleman and falcon ${ }^{81}$, to identify the burst of CR1-E1.

\section{Sex-linked gene analyses}


523 After removing LTR-derived genes, we used BLAT ${ }^{82}$ to align the annotated coding sequences of

$524 \mathrm{~W}$-linked genes to the $Z$ chromosome to search for their gametologous pairs. Then we produced

525 pairwise gametolog alignments using MUSCLE, ${ }^{83}$ and then manually inspected the alignments

526 to remove genes with short or ambiguous alignments. For species other than the BOPs, gene

527 models of the $\mathrm{W}$ chromosomes were directly retrieved from the RefSeq genome annotation,

528 with some of them subjected to manual inspections. To determine the orthologous relationship

529 among the studied species, we first extracted the sequence of the longest protein of each gene.

530 Those protein sequences were subjected to all-vs-all BLAST search that was implemented

531 through the program proteinortho ${ }^{84}$. BLAST hits with identity lower than $50 \%$ or alignment

532 coverage lower than $50 \%$ were removed. We also took gene synteny information into account

533 when grouping orthologous genes. Besides the twelve female genomes for which we studied

534 the sex chromosomes, we also included high-quality genomes of great tit, hooded crow and

535 ostrich (Supplementary Table 9). We retained those orthologous groups if they contained

536 sequences of at least ten species.

To estimate the substitution rates of coding sequences, first we performed multiple sequence alignments for orthologous genes. We used the guidance2 pipeline (http://guidance.tau.ac.il/ver2/source.php) which employs PRANK to align sequences of codons.

540 To filter low-quality sites in the alignments, we ran trimal (http://trimal.cgenomics.org/) to further

541 filter the ambiguous alignments with '-gt 0.8'. The phylogeny of the birds was extracted from

542 Jetz et al. $(2012)^{85}$. We used codeml from the PAML package ${ }^{86}$ to estimate the synonymous

543 substitution rates (dS) and non-synonymous substitution rates (dN). To estimate chromosome-

544 wide $\mathrm{dN}$ and $\mathrm{dS}$, the sums of synonymous or nonsynonymous substitutions were divided by

545 those of the number of synonymous or nonsynonymous sites, as applied in Wright et al. ${ }^{19}$.

546 Individual genes with abnormal $\mathrm{dN}$ (higher than 0.1 , in total 179 genes) or dS (higher than 0.8 ,

547 in total 135 genes) out of 111,748 orthologous gene groups were removed. Confidence intervals

548 were calculated by 100 bootstraps. The GC content of the third position codons (GC3) was

549 calculated using codonW $\mathrm{W}^{87}$ for the longest isoform of each gene. Chromosome-wide $\mathrm{dN} / \mathrm{dS}(\omega)$

550 was calculated using the ratios of chromosome-wide $\mathrm{dN}$ to chromosome-wise $\mathrm{dS}$. The fast-Z

551 effect is measured by $Z / A$ value, the ratio of $\omega$ values of Z-linked genes to autosomal genes,

552 and we calculated the Z/A value for every terminal branch and internal branch. To determine

553 whether the difference of $\omega$ between Z-linked and autosomal genes wasis significant, we

554 performed permutation test by resampling 1000 times. The genes of chromosome 4 and

555 chromosome 5 were used to represent autosomal genes as the sizes of those two

556 chromosomes are similar to the $\mathrm{Z}$ chromosome. 
For each gametologous pair, we grouped together Z-linked genes and assembled Wlinked genes and performed multiple sequence alignment. The same guidance2 pipeline was used as in sequence divergence analysis. For S3 genes, we also included rifleman ${ }^{36}$. We used IQTREE ${ }^{88}$ to construct maximum likelihood phylogenetic trees. The best substitution model was automatically selected in by IQ-TREE. We ran 100 bootstraps to evaluate the confidence levels of phylogenies. Ostrich was used as the outgroup to root the tree. The gene ontology (GO) term annotations for both gametolog-pairs genes (list) and entire Z-linked genes (background) of chicken were analyzed using DAVID $6.8^{89}$. GO term enrichment was analyzed by comparing the number of appearance of GO terms of 'list' gene versus 'background' gene.

\section{Gene loss analysis}

568 We identified a total of 673 Z-linked orthologous genes that are shared between chicken and emu as the putative ancestral genes on the proto-sex chromosomes of birds. For the gene cluster that was lost in chicken at the DCC locus of S3, an ancestral gene content was inferred based on Fig. 3 of Patthey et al. ${ }^{53}$. They were then grouped into four evolutionary strata according to the strata annotation of songbird $Z$ chromosomes. At each node of the avian phylogenetic tree, we calculated the ratio of the number of lost genes to the number of ancestral genes at that node. For the nodes leading to Passerida and Corvida, if there were at least one species retaining a $\mathrm{W}$-linked gene, we inferred that this gene was present in their ancestor. Similarly, we defined the presence of ancestral genes in Passeriformes, Neoaves and Neognathae using other published avian W-linked gene information ${ }^{20,21}$.

\section{Gene expression analysis}

580 We downloaded the raw RNA-seq reads of green anole (brain, gonad, liver, heart and kidney)

581 and emu (brain, gonad and spleen) from SRA (Supplementary Table 9). In addition, we

582 collected transcriptomes of adult emu kidneys of both sexes. We used the RSEM pipeline ${ }^{90}$ to 583 quantify the gene expression levels. We used STAR ${ }^{91}$ to map raw reads to the transcriptomes

584 which was constructed based on gene annotations. The expression levels were estimated at the 585 gene level, in the form of TPM (Transcripts Per Million). The mean TPM value of biological 586 replicates was calculate for each gene. Tissue specificity of gene expression was estimated by 587 calculating tau ${ }^{92}$.

\section{Data availability}


Genome sequencing and RNA-seq data, and genome assemblies generated in this study have been deposited in the NCBI SRA under PRJNA491255.

\section{Acknowledgements}

594 We thank Australian National Wildlife Collection, CSIRO Sustainable Ecosystems (Leo Joseph), 595 Museum Victoria, Australia (Joanna Sumner), Division of Vertebrate Zoology Yale University,

596 Peabody Museum of Natural History (Kristof Zyskowski) for tissue samples; and Edwin Scholes

597 for discussions on birds-of-paradise. We also acknowledge the support from Science for Life

598 Laboratory, the National Genomics Infrastructure (NGI), Uppmax. L.X. is supported by the 599 uni:doc PhD. fellowship from University of Vienna. M.I. is supported by the Swedish Research 600 Council (grant number 621-2014-5113). A.S. is supported by the Swedish Research Council 601 (grant number 2016-05139) and thanks Muhammad Bilal for help with repeat annotations. Q.Z. 602 is supported by National Natural Science Foundation of China (31722050, 31671319), the 603 Thousand Talents Plan, the Fundamental Research Funds for the Central Universities, and 604 start-up funds from Zhejiang University. The computational analyses were performed on CUBE 605 cluster from Dept. of Computational System Biology of University of Vienna and Vienna 606 Scientific Cluster.

609 Figure 1. The $Z$ and $\mathbf{W}$ chromosomes of different songbirds.

610 A) We use medium ground finch as an example to demonstrate our identification and 611 verification of sex-linked sequences. For each scaffold shown as a circle with scaled size to its 612 length, the ratio of nucleotide sites that were mapped by male vs. female genomic reads is 613 plotted against the sequencing depth of this scaffold. Scaffold sequences are clustered 614 separately by their derived chromosomes with W-linked (red circles) and Z-linked (blue circles) 615 sequences showing the expected half the autosome (green) sequencing depth, and W-linked 616 sequences showing almost no mappable sites from male reads. B) The lengths of $Z$ and $W$ 617 chromosomes across the studied songbird species. The shorter length of raggiana BOP W 618 chromosome is probably caused by the low sequencing coverage. Species name marked in 619 blue are those that have male reads available for verifying the female-specificity of W-linked 620 sequences.

621

\section{Figure 2. Evolution strata of songbirds.}


623 A) Genomic synteny of the avian Z chromosomes. Each color represents one evolutionary

624 stratum of songbirds which does not apply to chicken or emu, as they have independent origins

625 of evolutionary strata except for S0. The location of DMRT1, the avian male-determining gene is

626 marked by the red dashed line. Generally, the synteny is more conserved in younger strata

627 between species. B) We use Lawes's parotia as an example to demonstrate the pairwise

628 sequence similarity pattern of evolutionary strata. The size of circles is scaled to the length of

629 sequence alignments between Z/W chromosomes. C) Transposable elements (LINEs and LTRs)

630 are more strongly enriched in older strata (S0 is the first stratum) except for LINEs at S3. Levels

631 of significance comparing neighboring strata are tested by Wilcoxon test and shown with

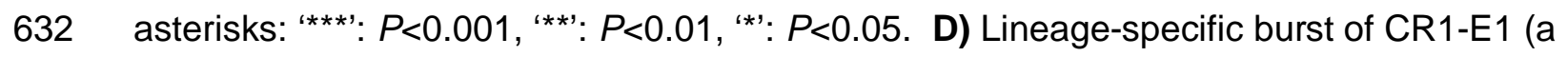

633 subfamily of CR-1 LINEs, red line) at the boundary of the PAR and S3 in songbirds, since their

634 divergence with other passerine species. Other subfamilies of CR1 elements are also plotted

635 with the green line for comparison. E) Phylogenetic tree using Z- and W-linked gametolog

636 sequences of the gene C18orf25 located at S3. Lineages are clustered by chromosomes (red or

637 blue), not by species, suggesting S3 independently formed in rifleman, chicken and the ancestor

638 of songbirds.

Figure 3. Fast-Z evolution of songbirds.

641 We show the difference of evolutionary rates between Z-linked genes vs. autosomal genes (Z/A

642 value), as a measurement of fast- $Z$ effect throughout the lineages of studied songbird species.

643 The tree length and color is scaled to the Z/A value, with lineages that show a significant

644 (permutation test, $P<0.05$ ) fast-Z pattern labelled with asterisks. '***': $P<0.001$, “**': $P<0.01$, ‘*':

$645 P<0.05$. We also labelled their information mating systems ('monogamy' vs. 'polygamy'), and

646 male display type ${ }^{54}$ ('lekking', 'exploded lekking', 'solitary display').

648 Figure 4. W-linked genes are preserved by purifying selection.

649 A) We show the retained W-linked genes of each studied songbird species, as well as those of

650 chicken, with homologous genes aligned vertically. The order of genes follows that of their emu

651 homologs along the $Z$ chromosome. The colors represent the evolutionary strata among

652 songbirds. B) The Z-linked genes without W-linked homologs (green, 'Lost') evolve faster than

653 those with W-linked homologs retained (red, 'Retained'), as indicated by their branch lengths

654 scaled to dN/dS ratios. C) The Z-linked genes whose W-linked homologs have become lost

655 (upper panel) tend to have a higher expression level (measured by TPM) in their lizard orthologs

656 than those with $\mathrm{W}$-linked homologs retained (lower panel). The genes are divided further by the 
657 stratum they reside on, and the expression level is shown by log-transformed medium

658 expression values of each category as color-coded heatmap. D) Gene expression tissue

659 specificity in green anole lizard for the homologous avian Z-linked genes.

Figure 5. Comparison of gene loss between $W$ chromosomes of songbirds and $Y$

662 chromosomes of primates.

663 A) We show the percentage of gene loss, and ancestral gene number for each evolutionary

664 stratum at each phylogenetic node. B) Similar analyses for the Y-linked gametologs of primates

665 based on the data of ${ }^{21}$, with $\mathrm{S} 1$ as the first stratum of eutherian mammals. C) We show the

666 length of songbird $W$ or primate $Y$ chromosomes scaled to the ancestral gene number of each

667 evolutionary stratum, with the color scaled to the overall percentage of gene loss. The ages of

668 evolutionary strata are indicated by the number (in millions of years) at the nodes below the

669 bars. As eutherian mammals have much larger ancestral evolutionary strata than those of birds,

670 they probably suffer a more severe gene loss on the $\mathrm{Y}$ chromosome.

\section{Reference}

673 1. IOC World Bird List 8.2.

674 2. Barker, F.K., Cibois, A., Schikler, P., Feinstein, J. \& Cracraft, J. Phylogeny and

675

676

677

678

679

680

681

682

683

684

685

686

687

688

689

690

691

692

693

694

695

696

697 diversification of the largest avian radiation. Proc Natl Acad Sci U S A 101, 11040-5 (2004).

3. Ellegren, $\mathrm{H}$. et al. The genomic landscape of species divergence in Ficedula flycatchers. Nature 491, 756-760 (2012).

4. Poelstra, J.W. et al. The genomic landscape underlying phenotypic integrity in the face of gene flow in crows. Science 344, 1410-1414 (2014).

5. Tuttle, E.M. et al. Divergence and functional degradation of a sex chromosome-like supergene. Curr Biol 26, 344-50 (2016).

6. Laine, V.N. et al. Evolutionary signals of selection on cognition from the great tit genome and methylome. Nat Commun 7, 10474 (2016).

7. Mank, J.E. Sex chromosomes and the evolution of sexual dimorphism: lessons from the genome. Am Nat 173, 141-50 (2009).

8. Coyne, J.A. Genetics and speciation. Nature 355, 511 (1992).

9. Charlesworth, B., Coyne, J. \& Barton, N. The relative rates of evolution of sex chromosomes and autosomes. Am Nat 130, 113-146 (1987).

10. Smeds, L. et al. Evolutionary analysis of the female-specific avian W chromosome. Nat. Commun. 6, 7330 (2015).

11. Hooper, D.M. \& Price, T.D. Chromosomal inversion differences correlate with range overlap in passerine birds. Nat Ecol Evol 1, 1526-1534 (2017).

12. Backström, N. et al. A high-density scan of the $Z$ chromosome in Ficedula flycatchers reveals candidate loci for diversifying selection. Evolution 64, 3461-3475 (2010).

13. Elgvin, T.O. et al. Hybrid speciation in sparrows II: a role for sex chromosomes? Mol Ecol 20, 3823-37 (2011). 
698

699

700

701

702

703

704

705

706

707

708

709

710

711

712

713

714

715

716

717

718

719

720

721

722

723

724

725

726

727

728

729

730

731

732

733

734

735

736

737

738

739

740

741

742

743

744

745

746

747

748

14. Storchová, R., Reif, J. \& Nachman, M.W. Female heterogamety and speciation: reduced introgression of the $\mathrm{Z}$ chromosome between two species of nightingales. Evolution 64, 456-471 (2010).

15. Ellegren, $\mathrm{H}$. et al. The genomic landscape of species divergence in Ficedula flycatchers. Nature 491, 756-60 (2012).

16. Li, W.H., Yi, S. \& Makova, K. Male-driven evolution. Curr Opin Genet Dev 12, 650-6 (2002).

17. Vicoso, B. \& Charlesworth, B. Effective population size and the faster-X effect: an extended model. Evolution 63, 2413-26 (2009).

18. Mank, J.E., Nam, K. \& Ellegren, H. Faster-Z evolution is predominantly due to genetic drift. Mol Biol Evol 27, 661-670 (2010).

19. Wright, A.E. et al. Variation in promiscuity and sexual selection drives avian rate of Faster-Z evolution. Mol Ecol 24, 1218-35 (2015).

20. Zhou, Q. et al. Complex evolutionary trajectories of sex chromosomes across bird taxa. Science 346, 1246338 (2014).

21. Bellott, D.W. et al. Avian W and mammalian $Y$ chromosomes convergently retained dosage-sensitive regulators. Nat Genet 49, 387-394 (2017).

22. Charlesworth, D., Charlesworth, B. \& Marais, G. Steps in the evolution of heteromorphic sex chromosomes. Heredity 95, 118-28 (2005).

23. Bachtrog, D. Y-chromosome evolution: emerging insights into processes of $Y$ chromosome degeneration. Nat Rev Genet 14, 113-24 (2013).

24. Soh, Y.Q. et al. Sequencing the mouse $Y$ chromosome reveals convergent gene acquisition and amplification on both sex chromosomes. Cell 159, 800-13 (2014).

25. Charlesworth, B. \& Charlesworth, D. The degeneration of $Y$ chromosomes. Philos Trans $R$ Soc Lond B Biol Sci 355, 1563-72 (2000).

26. Bachtrog, D. The temporal dynamics of processes underlying $\mathrm{Y}$ chromosome degeneration. Genetics 179, 1513-25 (2008).

27. Bellott, D.W. et al. Mammalian Y chromosomes retain widely expressed dosagesensitive regulators. Nature 508, 494-499 (2014).

28. Zhou, Q. \& Bachtrog, D. Sex-specific adaptation drives early sex chromosome evolution in Drosophila. Science 337, 341-345 (2012).

29. Koerich, L.B., Wang, X., Clark, A.G. \& Carvalho, A.B. Low conservation of gene content in the Drosophila Y chromosome. Nature 456, 949-51 (2008).

30. Smeds, L. et al. Evolutionary analysis of the female-specific avian W chromosome. Nat Commun 6, 7330 (2015).

31. Cortez, D. et al. Origins and functional evolution of $Y$ chromosomes across mammals. Nature 508, 488-93 (2014).

32. Bergero, R., Forrest, A., Kamau, E. \& Charlesworth, D. Evolutionary strata on the X chromosomes of the dioecious plant Silene latifolia: evidence from new sex-linked genes. Genetics 175, 1945-1954 (2007).

33. Lahn, B.T. \& Page, D.C. Four evolutionary strata on the human X chromosome. Science 286, 964-7 (1999).

34. Gorelick, R. et al. Abrupt shortening of bird W chromosomes in ancestral Neognathae. Biol. J. Linn. Soc. Lond. 119, 488-496 (2016).

35. Rice, W.R. The accumulation of sexually antagonistic genes as a selective agent promoting the evolution of reduced recombination between primitive sex chromosomes. Evolution 41, 911-914 (1987).

36. Zhang, G. et al. Comparative genomics reveals insights into avian genome evolution and adaptation. Science 346, 1311-1320 (2014).

37. Frankl-Vilches, C. et al. Using the canary genome to decipher the evolution of hormonesensitive gene regulation in seasonal singing birds. Genome Biol 16, 19 (2015). 
749

750

751

752

753

754

755

756

757

758

759

760

761

762

763

764

765

766

767

768

769

770

771

772

773

774

775

776

777

778

779

780

781

782

783

784

785

786

787

788

789

790

791

792

793

794

795

796

797

38. Qu, Y. et al. Ground tit genome reveals avian adaptation to living at high altitudes in the Tibetan plateau. Nat Commun 4, 2071 (2013).

39. Elgvin, T.O. et al. The genomic mosaicism of hybrid speciation. Sci Adv 3, e1602996 (2017).

40. Claramunt, S. \& Cracraft, J. A new time tree reveals Earth historys imprint on the evolution of modern birds. Science Advances 1, e1501005-e1501005 (2015).

41. Smeds, L. et al. Genomic identification and characterization of the pseudoautosomal region in highly differentiated avian sex chromosomes. Nat Commun 5, 5448 (2014).

42. Pala, I. et al. Evidence of a neo-sex chromosome in birds. Heredity 108, 264-72 (2012).

43. Suh, A., Smeds, L. \& Ellegren, H. Abundant recent activity of retrovirus-like retrotransposons within and among flycatcher species implies a rich source of structural variation in songbird genomes. Mol Ecol 27, 99-111 (2018).

44. Kapusta, A. \& Suh, A. Evolution of bird genomes-a transposon's-eye view. Ann $N Y$ Acad Sci 1389, 164-185 (2017).

45. Gorelick, R. et al. Abrupt shortening of bird W chromosomes in ancestral Neognathae. Biological Journal of the Linnean Society 119, 488-496 (2016).

46. Ross, M.T. et al. The DNA sequence of the human X chromosome. Nature 434, 325-37 (2005).

47. Poelstra, J.W. et al. The genomic landscape underlying phenotypic integrity in the face of gene flow in crows. Science 344, 1410-4 (2014).

48. Wright, A.E., Dean, R., Zimmer, F. \& Mank, J.E. How to make a sex chromosome. Nat Commun 7, 12087 (2016).

49. Duret, L. \& Galtier, N. Biased gene conversion and the evolution of mammalian genomic landscapes. Annu Rev Genomics Hum Genet 10, 285-311 (2009).

50. Kent, T.V., Uzunović, J. \& Wright, S.I. Coevolution between transposable elements and recombination. Phil Trans $R$ Soc B 372, 20160458 (2017).

51. Suh, A. et al. Mesozoic retroposons reveal parrots as the closest living relatives of passerine birds. Nat Commun 2, 443 (2011).

52. Friocourt, F. et al. Recurrent DCC gene losses during bird evolution. Sci Rep 7, 37569 (2017).

53. Patthey, C., Tong, Y.G., Tait, C.M. \& Wilson, S.I. Evolution of the functionally conserved DCC gene in birds. Sci Rep 7, 42029 (2017).

54. Irestedt, M., Jonsson, K.A., Fjeldsa, J., Christidis, L. \& Ericson, P.G. An unexpectedly long history of sexual selection in birds-of-paradise. BMC Evol Biol 9, 235 (2009).

55. Diamond, J. Biology of birds of paradise and bowerbirds. Ann Rev Ecol Syst 17, 17-37 (1986).

56. Kirkpatrick, M. \& Ryan, M.J. The evolution of mating preferences and the paradox of the lek. Nature 350, 33-38 (1991).

57. Suh, A. The Specific Requirements for CR1 Retrotransposition Explain the Scarcity of Retrogenes in Birds. J. Mol. Evol. 81, 18-20 (2015).

58. Stock, M. et al. Ever-young sex chromosomes in European tree frogs. PLoS Biol 9, e1001062 (2011).

59. Vicoso, B., Emerson, J.J., Zektser, Y., Mahajan, S. \& Bachtrog, D. Comparative sex chromosome genomics in snakes: differentiation, evolutionary strata, and lack of global dosage compensation. PLoS Biol 11, e1001643 (2013).

60. Charlesworth, D. Evolution of recombination rates between sex chromosomes. Philos Trans $R$ Soc Lond B Biol Sci 372(2017).

61. Lemaitre, C. et al. Footprints of inversions at present and past pseudoautosomal boundaries in human sex chromosomes. Genome Biol Evol 1, 56-66 (2009). 
798

799

800

801

802

803

804

805

806

807

808

809

810

811

812

813

814

815

816

817

818

819

820

821

822

823

824

825

826

827

828

829

830

831

832

833

834

835

836

837

838

839

840

841

842

843

844

845

846

847

62. Knief, U. et al. Fitness consequences of polymorphic inversions in the zebra finch genome. Genome Biol 17, 199 (2016).

63. Campagna, L. et al. Repeated divergent selection on pigmentation genes in a rapid finch radiation. Sci Adv 3, e1602404 (2017).

64. Moghadam, H.K., Pointer, M.A., Wright, A.E., Berlin, S. \& Mank, J.E. W chromosome expression responds to female-specific selection. Proc Natl Acad Sci U S A 109, 820711 (2012).

65. Peona, V., Weissensteiner, M.H. \& Suh, A. How complete are "complete" genome assemblies?-An avian perspective. Mol Ecol Resour. 0.

66. Amundsen, T. Why are female birds ornamented? Trends Ecol Evol 15, 149-155 (2000).

67. Irwin, D.E. Sex chromosomes and speciation in birds and other ZW systems. Mol Ecol 27, 3831-3851 (2018).

68. Gnerre, S. et al. High-quality draft assemblies of mammalian genomes from massively parallel sequence data. Proc. Natl. Acad. Sci. U. S. A. 108, 1513-1518 (2011).

69. Luo, R. et al. SOAPdenovo2: an empirically improved memory-efficient short-read de novo assembler. Gigascience 1, 18 (2012).

70. Cantarel, B.L. et al. MAKER: an easy-to-use annotation pipeline designed for emerging model organism genomes. Genome Res 18, 188-196 (2008).

71. Korf, I. Gene finding in novel genomes. BMC Bioinformatics 5, 59 (2004).

72. Stanke, M. \& Waack, S. Gene prediction with a hidden Markov model and a new intron submodel. Bioinformatics 19, ii215-ii225 (2003).

73. http://www.repeatmasker.org.

74. Prost, S. et al. Genome analyses show strong selection on coloration, morphological and behavioral phenotypes in birds-of-paradise. bioRxiv, 287086 (2018).

75. Marçais, G. et al. MUMmer4: A fast and versatile genome alignment system. PLoS Comput Biol 14, e1005944 (2018).

76. $\mathrm{Li}, \mathrm{H}$. Aligning sequence reads, clone sequences and assembly contigs with BWA-MEM. (2013).

77. Singhal, S. et al. Stable recombination hotspots in birds. Science 350, 928-32 (2015).

78. Yazdi, H.P. \& Ellegren, H. A genetic map of ostrich Z chromosome and the role of inversions in avian sex chromosome evolution. Genome Biol Evol 10, 2049-2060 (2018).

79. Zhang, J., Li, C., Zhou, Q. \& Zhang, G. Improving the ostrich genome assembly using optical mapping data. GigaScience 4, 24 (2015).

80. Harris, R.S. The Pennsylvania State University (2017).

81. Zhan, $X$. et al. Peregrine and saker falcon genome sequences provide insights into evolution of a predatory lifestyle. Nat Genet 45, 563-6 (2013).

82. Hancock, J.M. BLAT (BLAST-like Alignment Tool). in Dictionary of Bioinformatics and Computational Biology (2004).

83. Edgar, R.C. MUSCLE: multiple sequence alignment with high accuracy and high throughput. Nucleic Acids Res 32, 1792-1797 (2004).

84. Lechner, M. et al. Proteinortho: detection of (co-)orthologs in large-scale analysis. BMC Bioinformatics 12, 124 (2011).

85. Jetz, W., Thomas, G.H., Joy, J.B., Hartmann, K. \& Mooers, A.O. The global diversity of birds in space and time. Nature 491, 444-448 (2012).

86. Yang, Z. PAML 4: phylogenetic analysis by maximum likelihood. Mol Biol Evol 24, 15861591 (2007).

87. Peden, J. http://codonw.sourceforge.net//culong.html.

88. Nguyen, L.-T., Schmidt, H.A., von Haeseler, A. \& Minh, B.Q. IQ-TREE: a fast and effective stochastic algorithm for estimating maximum-likelihood phylogenies. Mol Biol Evol 32, 268-274 (2015). 
848 89. Jiao, X. et al. DAVID-WS: a stateful web service to facilitate gene/protein list analysis.

849

850

851

852

853 Bioinformatics 28, 1805-1806 (2012).

90. Li, B. \& Dewey, C.N. RSEM: accurate transcript quantification from RNA-Seq data with

91. Dobin, A. et al. STAR: ultrafast universal RNA-seq aligner. Bioinformatics 29, 15-21 (2013).

92. Yanai, I. et al. Genome-wide midrange transcription profiles reveal expression level relationships in human tissue specification. Bioinformatics 21, 650-9 (2005). 
bioRxiv preprint doi: https://doi.org/10.1101/454843; this version posted October 29, 2018. The copyright holder for this preprint (which was not certified by peer review) is the author/funder, who has granted bioRxiv a license to display the preprint in perpetuity. It is made available under aCC-BY-NC-ND 4.0 International license.

857 Figure 1. The $\mathrm{Z}$ and $\mathrm{W}$ chromosomes of different songbirds.

A

$\bigcirc$ chr5 $\bigcirc$ chrZ $\bigcirc$ chrW

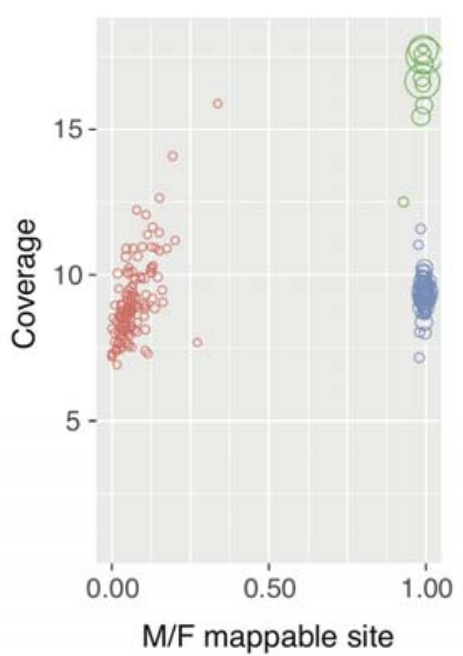

B

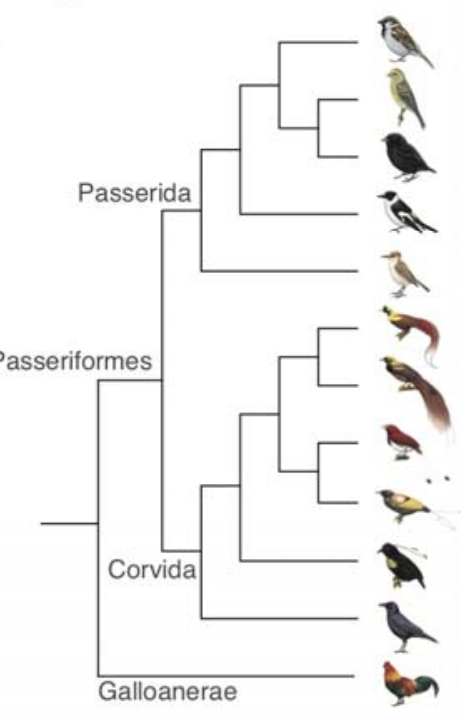

chrZ
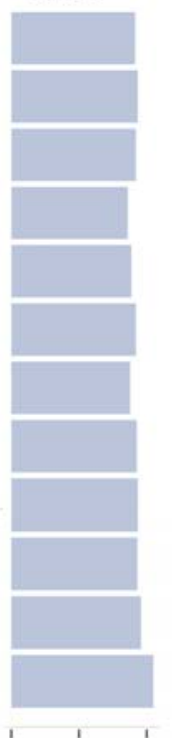

o $40 \quad 80$
chrW

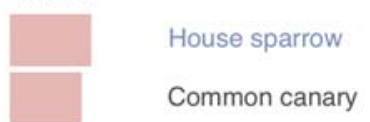

Medium ground finch

Collared flycatcher

Ground tit

Red BOP

Raggiana BOP

King BOP

Magnificant BOP

Lawes's parotia

American crow

Chicken 
bioRxiv preprint doi: https://doi.org/10.1101/454843; this version posted October 29, 2018. The copyright holder for this preprint (which was not certified by peer review) is the author/funder, who has granted bioRxiv a license to display the preprint in perpetuity. It is made available under aCC-BY-NC-ND 4.0 International license.

\section{A}

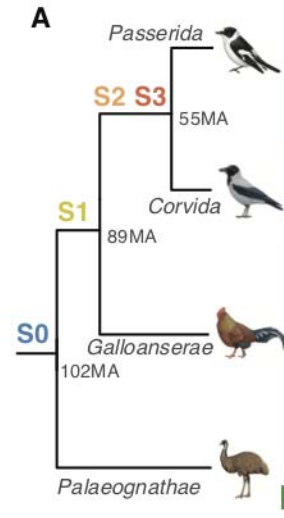

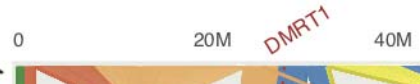

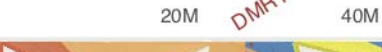

-
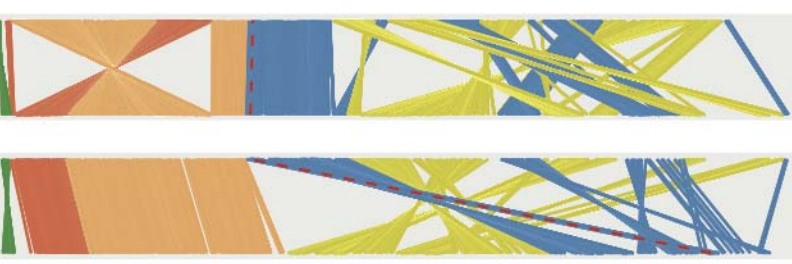

PAR S3

$80 \mathrm{M}$

$60 \mathrm{M}$

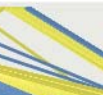

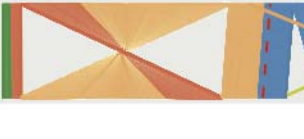

S2

S1

so
C

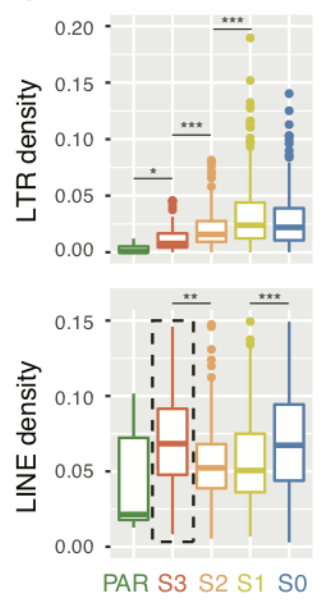

D

D

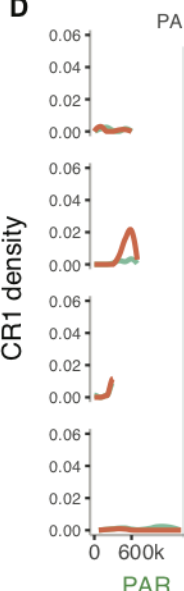 \\ 859 Figure 2. Evolutionary strata of songbirds.}

860
B Strata Os3 Os2 Os1 Oso

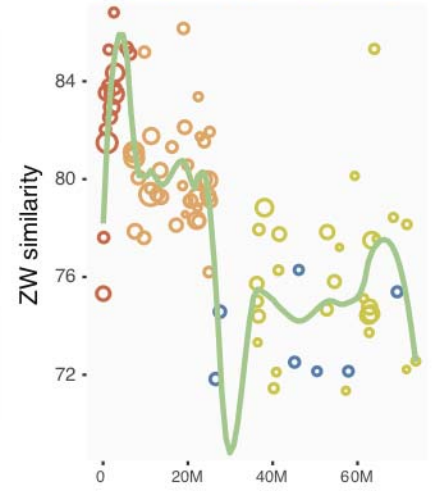

E

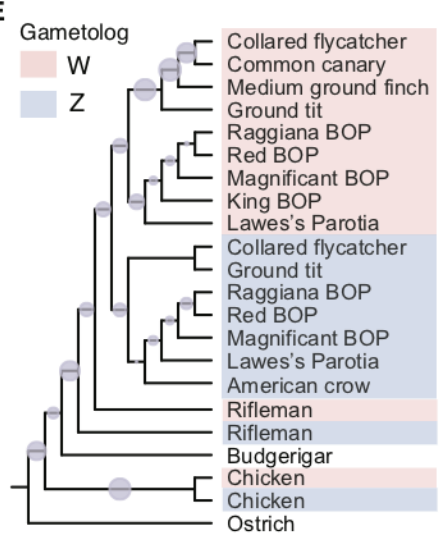

C18orf25 Bootstrapping $100 \quad 60$ 
bioRxiv preprint doi: https://doi.org/10.1101/454843; this version posted October 29, 2018. The copyright holder for this preprint (which was not certified by peer review) is the author/funder, who has granted bioRxiv a license to display the preprint in perpetuity. It is made available under aCC-BY-NC-ND 4.0 International license.

Figure 3. Fast-Z evolution of songbirds

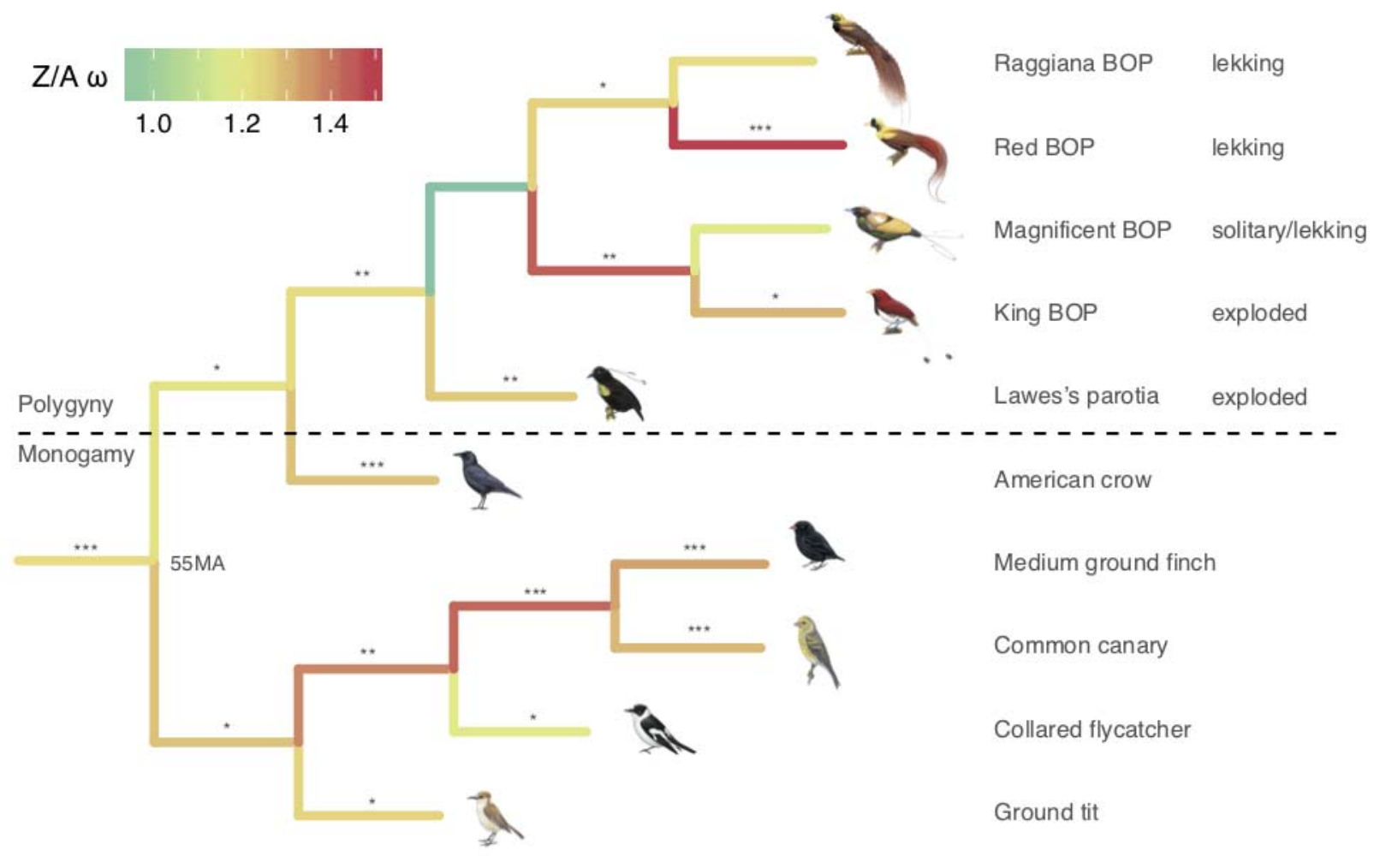


bioRxiv preprint doi: https://doi.org/10.1101/454843; this version posted October 29, 2018. The copyright holder for this preprint (which was not certified by peer review) is the author/funder, who has granted bioRxiv a license to display the preprint in perpetuity. It is made available under aCC-BY-NC-ND 4.0 International license.

Figure 4. W-linked genes are are preserved by purifying selection.

A \begin{tabular}{ll|l|l|l|l} 
Evolutionary strata & s3 & S2 & S1 & so
\end{tabular}

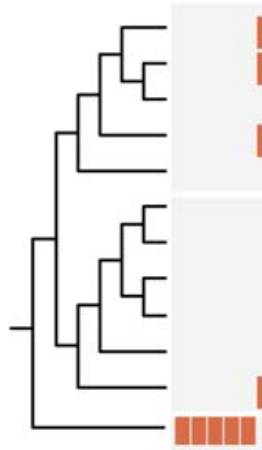

B

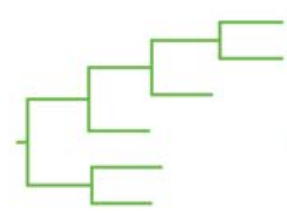

D

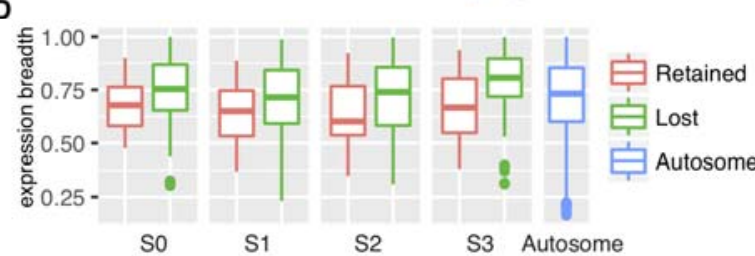

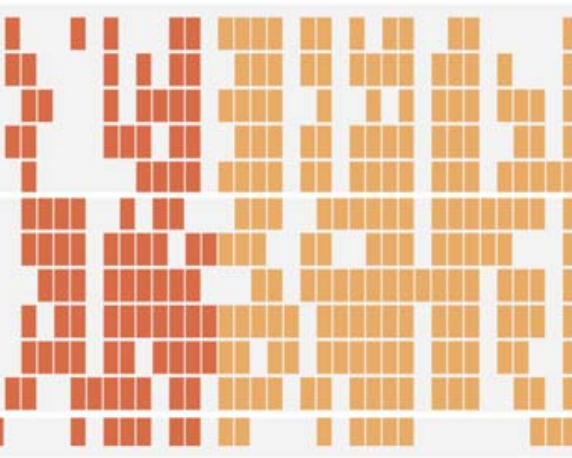

C

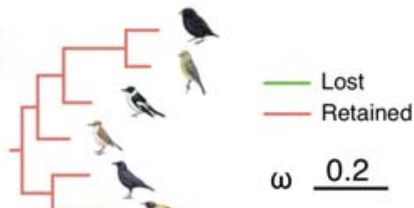

S3 Autosome

864

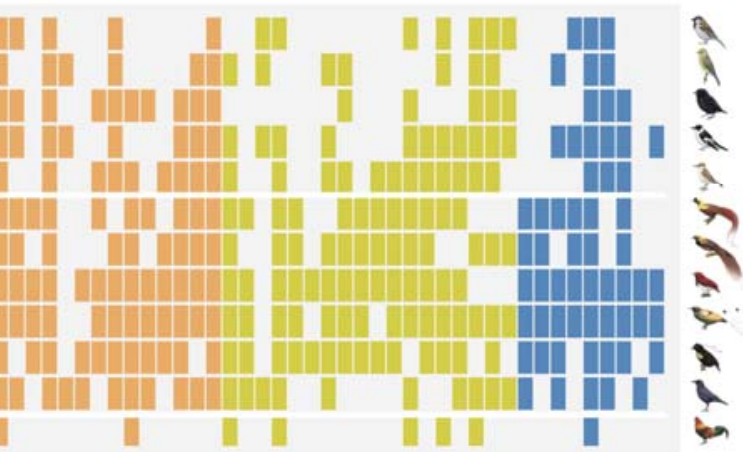

Gene expression

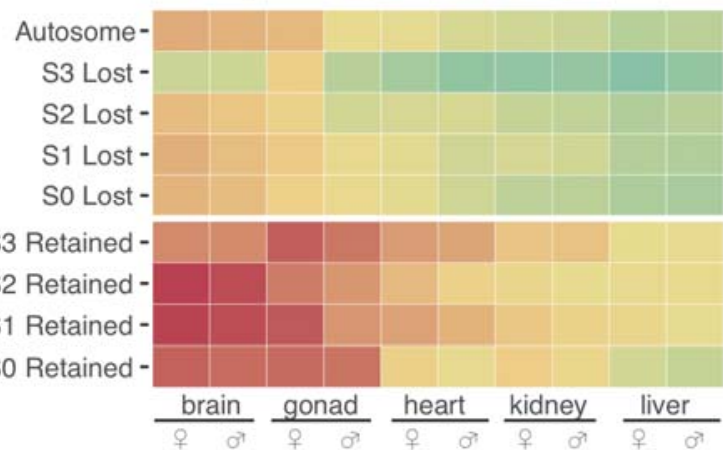


865 Figure 5. Comparison of gene loss between W chromosomes of songbirds and $Y$

866 chromosomes of primates.

A Songbird

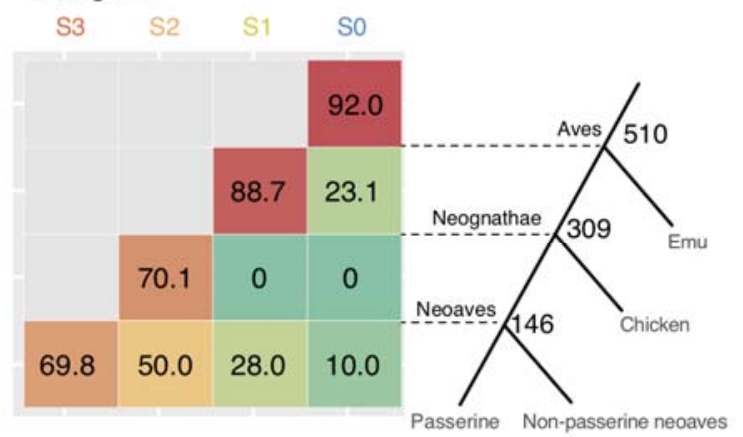

B
Primate

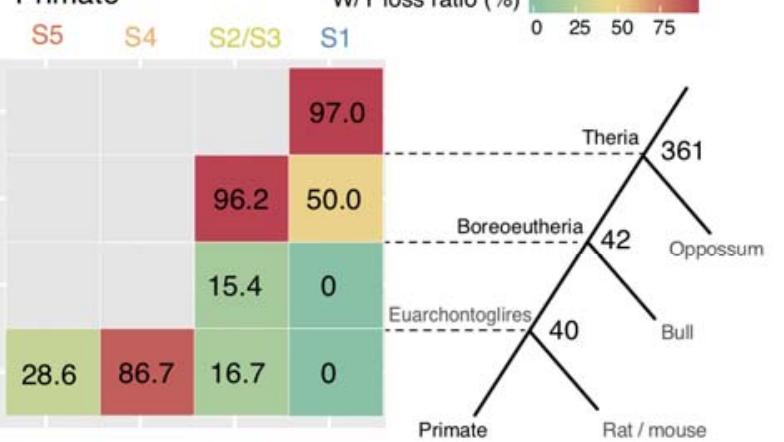

C

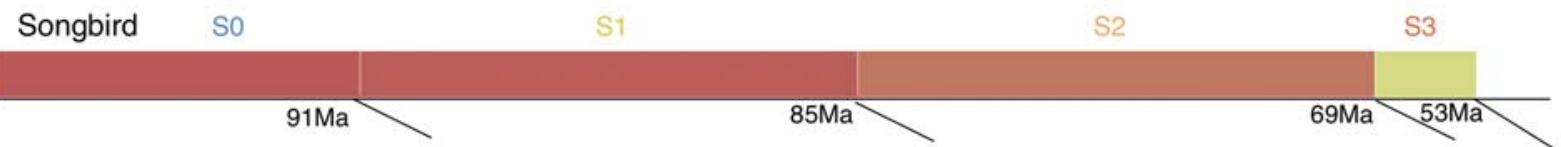

\begin{tabular}{|c|c|c|c|}
\hline Primate & S1 & $\mathrm{S} 2 / \mathrm{S}_{3}$ & S4 S5 \\
\hline & & & \\
\hline
\end{tabular}

